Discussion Paper No. 736

\title{
A COORDINATION GAME MODEL OF CHARITABLE GIVING AND SEED MONEY EFFECT
}

\author{
Kentaro Hatsumi
}

\author{
April 2009
}

Revised September 2009

The Institute of Social and Economic Research Osaka University

6-1 Mihogaoka, Ibaraki, Osaka 567-0047, Japan 


\title{
A Coordination Game Model of Charitable Giving and Seed Money Effect*
}

\author{
Kentaro Hatsumi ${ }^{\dagger}$ \\ First Version: December 24, 2007 \\ This Version: September 15, 2009
}

\begin{abstract}
If potential donors for a charity project possess the warm-glow properties in their preferences, we can represent their behavior with a coordination game. Accordingly, we construct a simultaneous incomplete information game model of charitable giving based on a simple global coordination game. We demonstrate that merely by the effect of seed money to shift the threshold requirement of the donations for project success downwards, the proportion of donors and the total amount of donations strictly and continuously increase with the amount of seed money. This result is compatible with the field experimental evidence in List and Lucking-Reiley [List, J. A., Lucking-Reiley, D., 2002. The Effects of Seed Money and Refunds on Charitable Giving: Experimental Evidence from a University Capital Campaign. Journal of Political Economy 110 (1), 215-233].
\end{abstract}

Keywords: charity, seed money, global game, coordination game, excludable threshold public good

JEL Classification Numbers: C72, D82, H41, L31

\footnotetext{
*I would like to thank Atsushi Kajii and Shigehiro Serizawa for their helpful suggestions. I am also grateful to Tadashi Sekiguchi, Daisuke Oyama, Kazuhiko Hashimoto, Shuhei Morimoto, as well as other participants at the 2009 Econometric Society Far East and South Asia Meeting, the 2009 Japanese Economic Association Spring Meeting, the 2008 Kyoto Game Theory Workshop and seminars at Osaka University for their helpful comments and discussions. The financial support from the Japan Society for the Promotion of Science via the Research Fellowship for Young Scientist is gratefully acknowledged.

${ }^{\dagger}$ Corresponding to: Institute of Social and Economic Research, Osaka University, 6-1, Mihogaoka, Ibaraki, 567-0047, Japan. Email: hatsumi at iser.osaka-u.ac.jp
} 


\section{Introduction}

\subsection{Charitable Giving and Coordination Game}

Why should economists study charity? We present two responses to this question. The first is the considerable impact of charitable behavior on the economy. For example, the Giving USA Foundation (2006) estimates that in the United States, total charitable donations in 2005 were $\$ 260.28$ billion, of which $\$ 199.07$ billion ( $76.5 \%$ of the total) comprised individual contributions. The size of these donations constitutes an important topic of research. The second is that charity is one of areas for which economists still do not possess a suitable theory. Andreoni (2006b) argues "philanthropy is one of the greatest puzzles for economics." Mainstream economics has assumed that people are extremely selfish and only interested in their selfbeneficial consumptions. Economists have constructed models based on this assumption. However, the assumption of selfishness is incompatible with charitable behavior. Even today, the field of philanthropy remains a puzzle and requires focused scholarly attention.

In recent years, it has been revealed that when considering charitable behavior, especially in large economies, the warm-glow property of preference is important. A preference with warm-glow property is typically represented by a utility function that possesses a three-dimensional domain: the private consumption, the total amount of donations from all contributors to a charity, and her own contribution to a charity. This contrasts with the classical preference represented by a utility function with only a two-dimensional domain: the private consumption, and the total amount of donations from all contributors to a charity. Warm-glow is the property that increases a donor's utility as a result of her own contribution. ${ }^{1}$ This property has been developed theoretically by Andreoni (1988, 1989, 1990), and supported empirically by studies such as Palfrey and Prisbrey $(1996,1997)$ and Ribar and Wilhelm (2002). ${ }^{2}$ In particular, Andreoni (1988) and Ribar and Wilhelm (2002) suggest that in a large economy, the warm-glow property is crucial to collect adequate amounts of charitable contributions.

A simple simultaneous game of a capital campaign for charity confirms their finding as follows. A capital campaign constitutes a funding drive for a charity that incurs a large fixed cost to ensure project success. ${ }^{3}$ A campaign to collect funds to build a hospital is a case in point. If the fixed cost for the project success is very large and there are many potential contributors, each contribution from each donor has almost no effect on project success. Assume a potential donor in a large economy has two choices Donate and Not Donate, and possesses a simple classical preference that depends on her

\footnotetext{
${ }^{1}$ Warm-glow is also called impure-altruism or joy-of-giving.

${ }^{2}$ Andreoni (2006b) contains a summary and discussions on the warm-glow property.

${ }^{3}$ We owe this definition of a capital campaign to Andreoni (1998).
} 
own private consumption and whether the project is a success or a failure. The latter condition is equivalent to whether the total amount of donations exceeds the fixed cost or not. Assume that even if the project fails, the donations are nonrefundable. Also assume that if a sufficiently large number of potential donors choose Donate, the project succeeds. Then her payoff is represented as follows.

\begin{tabular}{l|cc} 
& Success & Failure \\
\hline Donate & Happy & Unhappy \\
Not Donate & Very Happy & Neutral
\end{tabular}

Given that a potential donor cares only for her own private consumption and project success and she has no effect on project success, she is better off choosing Not Donate if the project succeeds. Of course, Not Donate also yields greater happiness if the project fails. From the Payoff Table (1), Not Donate is the strictly dominant strategy and each potential donor chooses this action. It is a prisoner's dilemma game and represents the classical free-riding problem.

However, if people possess the warm-glow property in their preferences, what is the payoff table in the same capital campaign game for a large number of potential donors? It is natural to consider that the payoff table is given as follows.

\begin{tabular}{l|cc} 
& Success & Failure \\
\hline Donate & Very Happy & Unhappy \\
Not Donate & Happy & Neutral
\end{tabular}

A contribution makes a donor with a warm-glow property happier than when not contributing if the project is a success. ${ }^{4}$ Then it has two pure strategy Nash equilibria; that is, either everybody chooses Donate, or everybody chooses Not Donate. This is a typical coordination game.

The coordination game is one of the most classical problems in game theory and has significantly improved in recent years due to Carlsson and van Damme (1993). They consider a simultaneous incomplete information coordination game where each agent has little knowledge about the type distribution, and they show the existence of a unique equilibrium with few assumptions concerning the strategies of agents. Even in the standard Bayesian coordination game where the type distribution is common knowledge, a unique equilibrium is difficult to obtain. Consequently, their work has attracted much attention. Their game model, known as the global game, has become popular with game theorists and has been extended in a number

\footnotetext{
${ }^{4}$ Ribar and Wilhelm (2002) find that in a large economy, the warm-glow property has a much stronger effect than the altruistic property concerned only with project success or failure in this example. Following this, the payoff to choose Not Donate with project success is even represented by Neutral.
} 
of different directions. Thanks to Morris and Shin $(1998,2003)$, it has now been extended to encompass a coordination problem in a large economy. ${ }^{5}$

If there are a sufficiently large number of potential donors with the warmglow property of preference, donation behavior for a charity project becomes a coordination game. In the situation of simultaneous donations, the incompleteness of information on the valuation of each potential donor for the charity project is also appropriate. These are the reasons why we consider that a global game is suitable for modeling charitable behavior. We construct a simultaneous incomplete information game model of charitable giving based on a simple global coordination game, and we characterize a unique equilibrium of the game. By means of comparative static analysis, we show that the model is compatible with the empirical studies of charitable behavior, especially the field experimental evidence in List and Lucking-Reiley (2002). We discuss this in the following subsection.

Finally, in this subsection, we note that this model has the same structure as the voluntary provision of an excludable threshold public good. If a contribution sufficient to exceed a threshold is necessary to produce a public good, and if the benefit for each contributor from the public good depends on how much she pays, then this has the same representation as the capital campaign to donors with the strong warm-glow property. ${ }^{6}$ Hence, the results in this paper also apply to the problem of producing an excludable threshold public good under incomplete information.

\subsection{Seed Money}

Seed money is a preliminary fund for charity publicly announced at the time when the project is announced. Leaders directly solicited to donate before the public announcement sometimes provide this seed money. Even before Andreoni (1998) introduced this problem to public economists, it was well known among charity fund-raisers that if seed money is granted, the donations from general contributors increase.

The field experimental evidence in List and Lucking-Reiley (2002) supports this knowledge among charity fund-raisers. ${ }^{7}$ List and Lucking-Reiley (2002) divide 3,000 potential donors into six groups. For three groups, each with 500 potential donors, they solicit the donation of a laboratory for environmental studies to purchase a computer at a fixed cost of $\$ 3,000$ with different levels of seed money. ${ }^{8}$ To the first group, they announce that the

\footnotetext{
${ }^{5}$ Well-known applications of the global game include debt pricing (Morris and Shin, 2004) and bank runs (Goldstein and Pauzner, 2005). Both are typical coordination problems.

${ }^{6}$ To represent the excludability of the public good in the Payoff Table (2), we need only change the payoff of choosing Not Donate with project success to Neutral.

${ }^{7}$ Other empirical studies on seed money include List and Rondeau (2003) and Potters et al. (2005).

${ }^{8}$ The other three groups of potential donors are assigned to an experiment concerning
} 
seed contribution is $\$ 300$, to the second group, $\$ 1000$, and to the third group, $\$ 2,000$. Table 1 provides the result. As shown, List and LuckingReiley (2002) clearly demonstrate that the total amount of contributions, the proportion of donors who actually contribute, and the per capita amount of contributions strictly and continuously increase according to the amount of seed money.

[Table 1 about here.]

By drawing on a global coordination game, our model demonstrates that the proportion of donors and the total amount of donations strictly and continuously increase with the amount of seed money. The result is compatible with List and Lucking-Reiley's (2002) field experimental work, and it supports the view that charitable giving actually involves a coordination game. The key to deriving this result is the threshold shift effect of seed money. This is the effect of seed money shifting the minimal requirement of donations for project success from the cost of the project itself to the amount of the cost minus the seed money. Merely because of this shift, for at least some proportion of potential donors, the expected payoff by donating becomes greater than when not donating. This behavior can be observed only under global game setting of incomplete information. In the standard Bayesian game where the type distribution is common knowledge, all potential donors often together decide to donate or not to donate in an equilibrium. This is inconsistent with the above empirical data by List and Lucking-Reiley (2002).

While our model is the first to capture the feature that the threshold shift by seed money causes a continuous increase in the proportion of donors and the total amount of donations, there is a considerable theoretical literature on the effect of seed money. Andreoni (1998) considers the seed money as a contribution from leaders, and He shows that the voluntary contribution from leaders cancels the zero-contribution equilibrium and leaves only the equilibrium with a positive amount of contributions. His discussion bases on a complete information game suitable to charitable giving among a small number of potential donors, while we construct a game with a large number of potential donors under incomplete information. Vesterlund (2003) and Andreoni (2006a) direct their attention to the quality signal effect of seed money. They explain that seed money donated by leaders indicates the high quality of the charity and therefore increases contributions from remaining donors. If there is a quality signal effect from seed money, it is easy to derive the strict increase in donations corresponding to the amount of seed

refunds. 
money. ${ }^{9}$ In contrast, our model shows that even without the quality signal effect, we can derive the positive effect of seed money. Indeed, the threshold shift effect and the quality signal effect are not contradictory but rather are complementary in increasing total contributions. Our study is meaningful even if we take the quality signal effect of seed money into account. ${ }^{10}$

\subsection{The Structure of the Paper}

The structure of the paper is as follows. Section 2 examines the basic model of charitable giving without seed money. In Subsection 2.1, we construct a model based on a simple global coordination game. We derive the equilibrium in Subsection 2.2, and the conditions for the efficiency and success of a project in Subsection 2.3. In Section 3, we consider the effect of seed money. In Subsection 3.1, we introduce seed money with the threshold shift effect, and we observe the impact on the behavior of potential donors. In Subsection 3.2, we calculate the minimal seed money needed to achieve efficiency and success. Section 4 discusses remaining inconsistencies with List and Lucking-Reiley (2002) and their conceivable solutions. Section 5 concludes. All proofs of propositions and remarks are in the Appendix.

\section{$2 \quad$ Basic Model and Results}

In this section, we present a model of charitable giving that provides a foundation for future comparative static analysis. The effect of seed money is not yet considered. We present the detailed game structure in Subsection 2.1, derive a unique equilibrium in Subsection 2.2, and provide remarks on the efficiency and success of the project in Subsection 2.3.

\subsection{Preliminaries}

There is a charity project and $n \in \mathbb{N}$ potential donors (agents). The project has a nonconvex technology. There exists a threshold level of its cost $C \in$ $\mathbb{R}_{++}$, and if the total provision from agents exceeds the threshold $C$, then the quality of the project is significantly improved. Imagine, for example, a charitable campaign to promote child health care in a rural area of a

\footnotetext{
${ }^{9}$ An interesting aspect of Vesterlund (2003) and Andreoni (2006a) is that the leaders donating the seed money and the amount of seed money donated are determined endogenously.

${ }^{10}$ There are several other studies on the effect of seed money. Romano and Yildirim (2001) show that in a two stage game of public good provision, the announcement of the contribution in the first stage sometimes brings about larger total contributions because of the warm-glow property. Bac and Bag (2003) consider a similar question about when it is beneficial for a charity fund-raiser to reveal the number of potential donors who are actually willing to donate. Bag and Roy (2008) explain the effect of seed money by the uncertainty regarding other donors' valuations of the project.
} 
developing country. If the charity collects sufficient donations to build a hospital, the quality of the project increases substantially. Refer to the project as a success (in the ex-post sense) if the donations collected from agents are sufficient to cover $C$, and a failure (in the ex-post sense) if the total donations are less than $C$.

We consider cases where the number of agents are sufficiently large. ${ }^{11}$ We adopt the assumption of a continuum of agents. To simplify the analysis, we normalize the length of the continuum of agents to 1 . Let $c=\frac{C}{n}$. Under the normalized setting, $c$ plays a role as the threshold cost instead of $C$. Thus we also refer to $c$ as the threshold cost.

The charity announces the threshold cost $c$ and solicits agents for donation. Through the announcement, each agent receives a signal of how worthy the project is in the case of both project success and failure. Based on this signal, each agent makes a decision about whether to donate. To simplify the analysis, we assume that her decision is merely a choice from her action set $\{$ Donate, Not Donate $\}$ and that the amount of donation on the basis of her signal is given exogenously. ${ }^{12}$

Let $v_{i} \in \mathbb{R}$ be the signal of the project value for an agent $i$ in the case of project success (ex-post sense). $v_{i}>0$ means that $i$ believes that the project value is positive and that it is worth donating a positive amount of money to if it succeeds. $v_{i} \leq 0$ means that she thinks that the project is not worthwhile and that donating money to it would be wasteful. In the case of $v_{i} \leq 0$, consider that the absolute value $\left|v_{i}\right|$ represents the degree of dislike for the project.

Assume that if $v_{i}>0$, she donates $x\left(v_{i}\right)$ when she makes a decision Donate, where $x: \mathbb{R}_{+} \rightarrow \mathbb{R}_{+}$is the same function among all agents, and possesses the properties of twice differentiability, and for all $v>0,0<$ $x(v)<v, x^{\prime}(v)>0$ and $x^{\prime \prime}(v) \leq 0($ i.e., $x(\cdot)$ is a weakly concave function). Furthermore, assume that $\lim _{v \rightarrow 0} x(v)=0, \lim _{v \rightarrow+\infty} x(v)=+\infty$ and there exists $\alpha \in(0,1)$ such that $\lim _{v \rightarrow 0} x^{\prime}(v)=\alpha$. Note that $v(\cdot)$ represents a wide class of functions including a linear function $\bar{x}: \mathbb{R}_{+} \rightarrow \mathbb{R}_{+}$such that for all $v>0, \bar{x}(v)=\alpha v$.

Moreover, assume that if $v_{i} \leq 0$, she donates $\epsilon>0$ if she decides to Donate. In the case of $v_{i} \leq 0$, it is natural to consider that in equilibria, she never decides to donate. The amount $\epsilon>0$ is a technical assumption. We assume throughout the paper that if the project is a failure, any agent $i$ believes that in the ex-post, the project is not attractive and the amount

\footnotetext{
${ }^{11}$ In each field experiment in List and Lucking-Reiley (2002), the number of potential donors (people who received solicitation letters) was 500.

${ }^{12}$ It is widely adopted as a simplification technique to limit the elements of each agent's action set to binary in the literature of global games. See, for example, Morris and Shin (2003). Moreover, even in classical models of discrete public good provision, such as Palfrey and Rosenthal (1984) and Gradstein (1994), the elements of the action set are binary limited to reduce complexity.
} 
of donation with a minus sign itself is her payoff.

Also assume that any agent has zero payoff if she does not donate, regardless of whether the project is a success or a failure. Because of the warm-glow property discussed earlier, when the project is a success, the payoff not to donate is smaller than that of donating. Thus we adopt the assumption of a zero payoff without donation for simplicity. Note that even if we allow some positive payoff for an agent who does not donate when the project is a success, the structure of the following investigation and the consequences are unchanged. ${ }^{13}$

The tables below summarize the payoffs.

If $v_{i}>0$,

\begin{tabular}{l|cc} 
& Success & Failure \\
\hline Donate & $v_{i}-x\left(v_{i}\right)$ & $-x\left(v_{i}\right)$ \\
Not Donate & 0 & 0
\end{tabular}

If $v_{i} \leq 0$,

\begin{tabular}{l|cc} 
& Success & Failure \\
\hline Donate & $v_{i}-\epsilon$ & $-\epsilon$ \\
Not Donate & 0 & 0
\end{tabular}

Based on these ex-post payoff tables, each agent is assumed to calculate her own von Neumann-Morgenstern expected interim payoff, and the to decide whether to donate.

Let us introduce the signal distribution structure and information setting for agents. Assume that the signal for each agent $v_{i}$ follows a normal distribution with mean $\theta$ and standard deviation $\sigma$ independently and identically. Given that the density function for a normal distribution approximates a wide class of symmetric density functions with a peak, this assumption is adequate. ${ }^{14}$ Let $F(\cdot ; \theta)$ denote the normal cumulative distribution function with mean $\theta$ and standard deviation $\sigma$, and let $f(\cdot ; \theta)$ denote its probability density function.

Following Morris and Shin (2003), we assume that it is common knowledge among agents that each signal $v_{i}$ for an agent $i$ is independently, identically, and normally distributed with standard deviation $\sigma$. However, each

\footnotetext{
${ }^{13}$ The following setting is also available. If the project is a success, an agent $i$ with $v_{i}>0$ who does not donate receives a payoff $b\left(v_{i}\right)$, where $b: \mathbb{R}_{+} \rightarrow \mathbb{R}_{+}$is a differentiable function such that for all $v>0,0 \leq b(v) \leq v-x(v), b^{\prime}(v) \geq 0,\{b(v) / x(v)\}^{\prime} \leq 0$, and there exists $\beta \in(0,1)$ with $\lim _{v \rightarrow 0} b^{\prime}(v)=\beta$. A detailed explanation is given in the Supplementary Note.

${ }^{14}$ In the field experiments in List and Lucking-Reiley (2002), all potential donors met two criteria: (i) the household's annual income was above $\$ 70,000$, and (ii) the household was known to have previously given to a charity. In this case, the standard deviation $\sigma$ is relatively small.
} 
agent $i$ has no information about the mean $\theta$. She has to predict the mean $\theta$ based on her realized signal $v_{i}$. We assume that her prior belief for $\theta$ is uniformly distributed over the real line $\mathbb{R}$. This prior belief is improper as the total probability mass is infinite; however, the posterior belief through Bayesian estimation is well defined. ${ }^{15}$ She believes, based on her signal $v_{i}$, that $\theta$ follows the normal distribution with mean $v_{i}$ and standard deviation $\sigma$. This is to say, from her point of view, $F\left(\cdot ; v_{i}\right)$ is the cumulative distribution function of the actual mean $\theta$, and $f\left(\cdot ; v_{i}\right)$ is its probability density function. It is an interesting aspect of this improper uniform prior belief that the same function is available to represent both the distribution of real signals and the distribution of the predictable mean based on the belief of a single agent.

In comparison with a standard Bayesian game wherein the signal distribution is common knowledge among agents, the assumption that the posterior belief of signal distribution for each agent is slightly different from those of others is quite natural. This is because of the following reasons. First, in a large economy where a continuum can approximate the total agents, if the distribution of signals is common knowledge, it almost represents a complete information game. This appears unrealistic. Second, especially when agents are relatively homogeneous (i.e., $\sigma$ is small) as in the field experiments in List and Lucking-Reiley (2002), it is reasonable that each agent predicts the signal distribution on the basis of her own signal.

A strategy for an agent $i$ is a function mapping her receiving signal $v_{i}$ to her action set $\{$ Donate, Not Donate $\}$. If both actions occasion the equivalent expected payoff for $i$, we assume that she selects Not Donate. This assumption is just to simplify the description, and the opposite assumption is, of course, available.

At this point, we define a Bayesian type of incomplete information game. In the next subsection, we consider the equilibrium in this game.

\subsection{The Equilibrium}

We are interested in Bayesian Nash equilibria of the game defined in the previous subsection. If possible, a unique equilibrium is preferable especially for the purpose of comparative statics. The global game analysis makes it possible to provide a unique equilibrium with a few natural additional assumptions to the strategies of agents. Furthermore, it is the strategy profile surviving the iterated elimination of interim strictly dominated strategies. Since this concept does not rely on the assumption that the strategies of other agents are common knowledge, it is a weaker condition than Bayesian Nash equilibrium.

\footnotetext{
${ }^{15}$ See Morris and Shin (2003) for a detailed explanation. They discuss the improper uniform prior belief with respect to the philosophy of Laplace.
} 
In this subsection, we characterize a unique strategy profile by the iterated elimination of interim strictly dominated strategies as the equilibrium. The equilibrium strategy possesses two interesting properties: symmetry and switching properties. A strategy is symmetric if all agents follow the same strategy. A strategy is a switching strategy around some cutoff point $k \in \mathbb{R}$ if there exists a unique cutoff point $k$ such that if an agent's receiving signal $v_{i}$ is above $k$, she donates; otherwise, she does not donate. A symmetric switching strategy $s: \mathbb{R} \rightarrow\{$ Donate, Not Donate $\}$ is formally written as follows.

$$
s\left(v_{i}\right)= \begin{cases}\text { Donate } & \text { if } v_{i}>k \\ \text { Not Donate } & \text { if } v_{i} \leq k\end{cases}
$$

Note that the symmetry and switching properties of the equilibrium are not assumptions but are obtained endogenously.

We introduce several functions that play important roles in the following investigation.

$$
\begin{gathered}
\int_{k}^{\infty} f(v ; \theta) d v \\
\int_{k}^{\infty} x(v) f(v ; \theta) d v
\end{gathered}
$$

(5) represents the proportion of agents whose signal is larger than $k$ when the mean signal is $\theta$. This function is useful no matter what strategies are adopted. (6) represents the total donation when the mean of the signals is $\theta$ and any agent receiving a signal larger than the cutoff point $k$ decides to donate. (6) is meaningful only when any agent adopts the switching strategy.

At the beginning of the investigation, notice the remark below.

Remark 1 . For any agent $i$ with $v_{i}<0$, Not Donate is the strictly dominant action.

This remark is straightforward from the payoff tables of the previous section, and it has an important role in the global game analysis. We require a similar property that for any agent $i$ with a sufficiently large signal $v_{i}$, Donate is the strictly dominant action.

Remember that we assume the number of agents to be large and approximated by a continuum of agents with length 1 . Therefore, an agent regards her contribution itself as zero even if she donates a positive amount of money. However, it is not natural that an agent with so large a signal that her own contribution covers the whole project cost $C$ (i.e., an agent $i$ with signal $v_{i}>0$ such that $x\left(v_{i}\right)>C$ ) would consider her contribution to be zero. Note that it is not the normalized cost $c$ but rather the original $C$. As her actual contribution covers the whole cost $C$, she has no reason to fear project failure. 
Assumption 1 below represents the above discussion. Let $V \in \mathbb{R}_{++}$be such that $x(V)=C$.

Assumption 1 . For any agent $i$ with $v_{i}>V$, Donate is the strictly dominant action. $^{16}$

We add one more assumption related to Assumption 1.

Assumption 2. $\int_{V}^{\infty} x(v) f(v ; \hat{\theta}(V)) d v \geq c$

Assumption 2 states that if the mean of signals is $\hat{\theta}(V)$ and agents with signals more than $V$ donate, the total donation from agents with signals larger than $V$ exceeds $c$ and the project is a success. As already discussed when we introduced Assumption 1, the signal $V$ is so large that $x(V)=C$. Given that $\hat{\theta}(V)$ is also large in accordance with $V$, it is also a natural assumption.

Assumptions 1 and 2 may appear somewhat outlandish. The existence of an agent $i$ with a high signal $v_{i}>C$ may be especially difficult to accept. However, we note that the model relies very little on these assumptions in two ways.

First, under the normal distribution of signals, the contribution of agents with signals larger than $\mathrm{C}$ is actually quite small. In fact, it is almost zero and relatively unimportant. What is important is not the existence of an agent $i$ with such a high signal $v_{i}>C$ but that of an agent $j$ with $v_{j}<v_{i}$ who believes that an $i$ with such a high signal $v_{i}>C$ exists. Alternatively, instead of $j$, we only need an agent $h$ with $v_{h}<v_{j}$ who thinks such $j$ exists. This type of expectation of a certain proportion of existing agents is the key to deriving the equilibrium in the global game analysis.

Second, if we assume that any agent takes a symmetric threshold strategy and this is common knowledge, then we can derive the same equilibrium as an interim Bayesian Nash equilibrium without Assumptions 1 and 2. See the Supplementary Note for more details.

Note that the interim payoff of an agent $i$ with a positive signal $v_{i}>0$ is represented by

$$
\operatorname{Pr}(\text { Success })\left(v_{i}-x\left(v_{i}\right)\right)+(1-\operatorname{Pr}(\text { Success }))\left(-x\left(v_{i}\right)\right) .
$$

Thus, agent $i$ decides to donate if

$$
\begin{aligned}
& (7)>0 \\
\Longleftrightarrow & \operatorname{Pr}(\text { Success })>\frac{x\left(v_{i}\right)}{v_{i}} .
\end{aligned}
$$

Since $\lim _{v_{i} \rightarrow 0} x\left(v_{i}\right)=0$ and $\lim _{v_{i} \rightarrow 0} x^{\prime}\left(v_{i}\right)=\alpha$, we have

$$
\lim _{v_{i} \rightarrow 0} \frac{x\left(v_{i}\right)}{v_{i}}=\alpha \quad \text { (by l'Hospital's rule.) }
$$

\footnotetext{
${ }^{16}$ It is important that an agent with a large enough signal has the strictly dominant action to donate. The value $V$ itself is not essential.
} 
Let a function $p: \mathbb{R}_{+} \rightarrow \mathbb{R}_{++}$be such that

$$
p\left(v_{i}\right)= \begin{cases}\frac{x\left(v_{i}\right)}{v_{i}} & \text { if } v_{i}>0 \\ \alpha & \text { if } v_{i}=0 .\end{cases}
$$

$p\left(v_{i}\right)$ represents the threshold probability such that $i$ with $v_{i}>0$ considers that if the probability of project success is larger than $p\left(v_{i}\right)$, Donate leads to a higher expected payoff than Not Donate. On the other hand, if the probability of project success is less than $p\left(v_{i}\right)$, Not Donate guarantees a higher expected payoff than Donate. The reason that $p(0)$ is defined as the limit value at $v_{i} \rightarrow 0$ is merely to simplify the following descriptions. Since $x(\cdot)$ is weakly concave and differentiable in $v_{i}>0$, we have the remark below.

Remark 2. $p(\cdot)$ is weakly decreasing and continuous in $v_{i} \geq 0$.

[Figure 1 about here.]

Let $\hat{\theta}: \mathbb{R}_{+} \rightarrow \mathbb{R}$ be a function such that for $v_{i} \geq 0$,

$$
\int_{\hat{\theta}\left(v_{i}\right)}^{\infty} f\left(v ; v_{i}\right) d v=p\left(v_{i}\right){ }^{17}
$$

See Figure 1 for an illustration. Remember that an agent $i$ with a signal $v_{i}$ believes the mean of normally distributed signals to be $v_{i}$ itself. Thus the equation represents that $i$ considers that the probability of the mean of signal distribution being larger than $\hat{\theta}\left(v_{i}\right)$ is $p\left(v_{i}\right)$ (i.e., $i$ considers $\operatorname{Pr}(\theta \geq$ $\left.\left.\theta\left(v_{i}\right)\right)=p\left(v_{i}\right)\right)$. Note that since $p(0)=\alpha \in(0,1), \hat{\theta}(0) \in \mathbb{R}$. Since $p(\cdot)$ is weakly decreasing in $v_{i} \geq 0$, we have the remark below.

Remark 3. (i) $\hat{\theta}\left(v_{i}\right)$ is strictly increasing and continuous in $v_{i} \geq 0$. (ii) For $v_{i}^{\prime}>v_{i} \geq 0, \hat{\theta}\left(v_{i}^{\prime}\right)-\hat{\theta}\left(v_{i}\right) \geq v_{i}^{\prime}-v_{i}$. (iii) $\lim _{v \rightarrow+\infty} \hat{\theta}(v)=+\infty$.

We introduce two more functions and discuss their properties.

$$
\int_{k}^{\infty} x(v) f\left(v ; \hat{\theta}\left(v_{i}\right)\right) d v
$$

(8) is a special case of (6), and the mean of signals is $\hat{\theta}\left(v_{i}\right)$. Since $\hat{\theta}\left(v_{i}\right)$ is strictly increasing in $v_{i} \geq 0$, the following remark is straightforward.

Remark 4. (8) is strictly increasing and continuous in $v_{i} \geq 0$ and strictly decreasing and continuous in $k \geq 0$.

\footnotetext{
${ }^{17}$ In the explicit form, $\hat{\theta}\left(v_{i}\right)=F^{-1}\left(1-p\left(v_{i}\right) ; v_{i}\right)$, where $F^{-1}\left(\cdot ; v_{i}\right)$ is the inverse function of $F\left(\cdot ; v_{i}\right)$. This expression is rarely used in the following investigation.
} 


$$
\int_{v_{i}}^{\infty} x(v) f\left(v ; \hat{\theta}\left(v_{i}\right)\right) d v
$$

(9) is a special case of (8). The mean of the signals is $\hat{\theta}\left(v_{i}\right)$ and the cutoff point $k=v_{i}$. Remark 3 induces the next remark.

Remark 5. (9) is strictly increasing and continuous in $v_{i} \geq 0$.

Let $G$ denote the Bayesian type of incomplete information game defined in Subsection 2.1 with Assumption 1 and 2. Now, we characterize a cutoff point and the equilibrium. Given a threshold cost $c$, let

$$
k^{*}= \begin{cases}0 & \text { if } \int_{0}^{\infty} x(v) f(v ; \hat{\theta}(0)) d v \geq c \\ \kappa & \text { otherwise }\end{cases}
$$

where $\kappa \in \mathbb{R}_{++}$satisfies

$$
\int_{\kappa}^{\infty} x(v) f(v ; \hat{\theta}(\kappa)) d v=c
$$

Note that since (9) diverges to infinity when $v_{i}$ goes to infinity (i.e., (9) $\rightarrow$ $\left.+\infty\left(v_{i} \rightarrow+\infty\right)\right), \kappa$ is uniquely determined if $\int_{0}^{\infty} x(v) f(v ; \hat{\theta}(0)) d v<c$.

Proposition 1. Let a symmetric switching strategy $s^{*}(\cdot)$ be such that

$$
s^{*}\left(v_{i}\right)= \begin{cases}\text { Donate } & \text { if } v_{i}>k^{*} \\ \text { Not Donate } & \text { if } v_{i} \leq k^{*}\end{cases}
$$

Then, in a game $G, s^{*}(\cdot)$ constitutes a unique strategy profile surviving the iterated elimination of interim strictly dominated strategies.

Note that from the definition of $k^{*}$, the actual mean of the signal distribution $\theta$ has no effect in determining the equilibrium cutoff point $k^{*}$. In contrast, $\hat{\theta}(\cdot)$ is quite important in determining $k^{*}$. The example below illustrates the proposition.

Example 1. Let a threshold level of $\operatorname{cost} c=6 .{ }^{18}$ Let the standard deviation of the signal distribution $\sigma=10$. Let for all $v_{i}>0, x\left(v_{i}\right)=\frac{1}{2} v_{i}$. Then, for all $v_{i}>0, p\left(v_{i}\right)=\frac{1}{2}$ and $\hat{\theta}\left(v_{i}\right)=v_{i}$. We calculate $k^{*}=4.02115$ and have Table 2 .

\section{[Table 2 about here.]}

\footnotetext{
${ }^{18}$ In each field experiment in List and Lucking-Reiley (2002), the threshold level of project cost for success is $\$ 3,000$ and the number of potential donors is 500 (i.e., $C=3,000$ and $n=500)$. In this case, $c=C / n=6$.
} 
Note that the proportion of actual contributors is represented by

$$
\int_{k^{*}}^{\infty} f(v ; \theta) d v
$$

and the total amount of donation is represented by

$$
\int_{k^{*}}^{\infty} x(v) f(v ; \theta) d v
$$

The mean amount of donation from actual contributors is $(11) /(10)$, and the project is a success if (11) is larger than $c$.

As in Example 1, the equilibrium cutoff point $k^{*}$ is often larger than 0 . This is because of a particular assumption of the global game that each agent possesses a different belief about the signal distribution. Under a standard Bayesian game model where the signal distribution and the strategies of other agents constitute common knowledge, all agents with signals larger than 0 decide together whether to donate or not depending on the actual mean of the signals $\theta$ in an equilibrium. Under the same setting as Example 1 , the standard Bayesian game analysis provides this in an equilibrium; if $\theta>4.02115$, agents with signals larger than 0 together decide to donate, and if $\theta \leq 4.02115$, any agent does not donate and the total amount of donation is equal to zero. This prediction is incompatible with the empirical research such as List and Lucking-Reiley (2002). It suggests the effectiveness of global game analysis in the study of charitable giving.

\subsection{Conditions for Efficiency and Success}

In this subsection, we first consider the condition that achieves the ex-post Pareto efficiency of the donation for agents. Next, we consider the condition for the success of the project.

We refer to a project as efficient if in the equilibrium, the action of agents derives ex-post Pareto efficiency. Formally, this is represented when $\int_{0}^{\infty} x(v) f(v ; \theta) d v \geq c$, any agent $i$ with signal $v_{i}>0$ chooses Donate, and any agent $j$ with signal $v_{j} \leq 0$ chooses Not Donate, otherwise, all agents select Not Donate. This is easily achievable in a equilibrium of the standard Bayesian Game discussed in the last paragraph of the previous subsection. However, it is more difficult to obtain it under the more realistic global game setting. In a game $G$, the following proposition holds.

Proposition 2. A project is efficient if and only if $k^{*}=0$ and $\int_{0}^{\infty} x(v) f(v ; \theta) d v \geq$ c.

Actually, this is equivalent to the condition for success when $k^{*}=0$. The intuition for this proposition is quite simple. Even though the project is a failure, some agents with signals larger than $k^{*}$ choose Donate. This 
implies that the project is not efficient. If the project is a success and $k^{*}>0$, an agent $i$ with $0<v_{i}<k^{*}$ chooses Not Donate, which brings about inefficiency. Therefore, the condition that occasions efficiency is only described in Proposition 2. if

As already mentioned in the previous subsection, a project is a success

$$
\int_{k^{*}}^{\infty} x(v) f(v ; \theta) d v \geq c
$$

This may be the most important matter for a charity fund-raiser. Note that if a project is efficient, then the project is a success. Besides, even in the case of $k^{*}>0$, the project is a success as the proposition below states.

Proposition 3. A project is a success if and only if it is efficient or $\theta \geq$ $\hat{\theta}\left(k^{*}\right)$.

If $k^{*}>0, \int_{k^{*}}^{\infty} x(v) f\left(v ; \hat{\theta}\left(k^{*}\right)\right) d v=c$. Thus $\theta \geq \hat{\theta}\left(k^{*}\right)$ induces $\int_{k^{*}}^{\infty} x(v) f(v ; \theta) d v \geq$ $c$ and the project succeeds.

In the next section, we introduce seed money. The main purpose for introducing seed money is to obtain the success or efficiency of a project. We show how to calculate the minimal amount of seed money for both efficiency and success.

\section{Seed Money}

We exogenously introduce seed money. We see the threshold shift effect of the seed money on the donation behavior of agents in Subsection 3.1. We also see how to obtain the success and the efficiency of the project by announcing seed money in Subsection 3.2.

\subsection{The Threshold Shift Effect}

Let $L \in[0, C)$ denote the amount of seed money. $L=0$ represents the state where no seed money is granted. If seed money $L$ is granted, the threshold level for project success shifts from $C$ to $C-L$. Assume that information about the project never changes except concerning the existence and amount of seed money. We consider that all other structures of the game except for the shift of the threshold for project success remain as before.

Let $\ell$ denote the normalized version of seed money; i.e., $\ell=\frac{L}{n}$. Consequently, the normalized version of the shifted threshold is $c-\ell$. We also rewrite the additional assumptions in Subsection 2.2 as follows. Let $V(\ell) \in \mathbb{R}_{++}$be such that $x(V(\ell))=C-L$.

Assumption 1'. For any agent $i$ with $v_{i}>V(\ell)$, Donate is the strictly dominant action.

Assumption 2'. $\int_{V(\ell)}^{\infty} x(v) f(v ; \hat{\theta}(V(\ell))) d v \geq c-\ell$. 
Let $G(\ell)$ denote a Bayesian game with the same structure as that in Subsection 2.1 with the shift of threshold level from $c$ to $c-\ell$ and Assumptions $1^{\prime}$ and 2'. Then we derive a unique equilibrium strategy $s^{*}(\cdot ; \ell)$ in a game $G(\ell)$ as a corollary of Proposition 1.

Corollary 1. In a game $G(\ell)$, a symmetric switching strategy $s^{*}(\cdot ; \ell)$ such that

$$
s^{*}\left(v_{i} ; \ell\right)= \begin{cases}\text { Donate } & \text { if } v_{i}>k^{*}(\ell) \\ \text { Not Donate } & \text { if } v_{i} \leq k^{*}(\ell),\end{cases}
$$

where

$$
k^{*}(\ell)= \begin{cases}0 & \text { if } \int_{0}^{\infty} x(v) f(v ; \hat{\theta}(0)) d v \geq c-\ell \\ \kappa(\ell) & \text { otherwise, }\end{cases}
$$

and $\kappa(\ell) \in \mathbb{R}_{++}$is such that

$$
\int_{\kappa(\ell)}^{\infty} x(v) f(v ; \hat{\theta}(\kappa(\ell))) d v=c-\ell
$$

constitutes a unique strategy profile surviving the iterated elimination of interim strictly dominated strategies.

Hereafter, we consider the case of $\int_{0}^{\infty} x(v) f(v ; \hat{\theta}(0)) d v<c$. Otherwise, not only success but also efficiency is obtained without seed money, and the introduction of seed money is then redundant. Let $\underline{\ell}=c-\int_{0}^{\infty} x(v) f(v ; \hat{\theta}(0)) d v$. According to Corollary 1, we have the remark below.

Remark 6. $k^{*}(\ell)$ is strictly decreasing and continuous in $\ell<\underline{\ell}$, and $k^{*}\left(\ell^{\prime}\right)=0$ for $\ell^{\prime} \geq \underline{\ell}$.

From Remark 6, we derive a proposition below that is quite compatible with the field experiments in List and Lucking-Reiley (2002).

Proposition 4. If a granted amount of seed money $\ell$ strictly increases, then both the proportion of agents who decide to donate and the total amount of donations strictly increase as long as $\ell<\underline{\ell}$.

The example below illustrates this proposition.

Example 2. Similarly to Example 1, let a threshold level of cost $c=6$, a standard deviation of signal distribution $\sigma=10$, and for all $v_{i}>0$, $x\left(v_{i}\right)=\frac{1}{2} v_{i}$. Consequently, we have the results in Table 3.

[Table 3 about here.] 
The mechanism behind Proposition 4 is quite simple and only depends on Remark 6. If $k^{*}(\ell)$ decreases, it is obvious that the number of agents with signals larger than the cutoff point $k^{*}(\ell)$ strictly increases, and then the proportion of agents who decide to donate and the total amount of donations also strictly increase. As discussed in the last part of Subsection 2.2 , the emergence of the cutoff point $k^{*}(\ell)$ strictly larger than 0 is due to the global game assumption that the actual signal distribution is not common knowledge but is noisily observed and guessed by each agent $i$ on the basis of her own signal $v_{i}$. This realistic setting of the global game is the key to the derivation of Proposition 4.

\subsection{Minimal Seed Money for Efficiency and Success}

The main purpose for introducing seed money is to obtain the efficiency or success of a project that is not achieved in its absence. Especially from the viewpoint of a charity fund-raiser, the attainment of project success is crucial. Even though we do not explicitly consider the cost of introducing seed money, charity fund-raisers actually require a considerable effort to collect seed money and are better off if the amount of seed money needed for efficiency or success is as small as possible. In this subsection, we derive the minimal amount of seed money to attain each of efficiency and success.

First, consider the minimal seed money for efficiency. Note that without seed money, the project is not efficient if $k^{*}(0)>0$ or $\int_{0}^{\infty} x(v) f(v ; \theta) d v<c$. In this case, we have the following proposition.

Proposition 5. The minimal amount of seed money for efficiency $\bar{\ell}$ is such that

$$
\bar{\ell}= \begin{cases}c-\int_{0}^{\infty} x(v) f(v ; \hat{\theta}(0)) d v & \text { if } \theta \geq \hat{\theta}(0) \\ c-\int_{0}^{\infty} x(v) f(v ; \theta) d v & \text { otherwise }\end{cases}
$$

This proposition is straightforward from Proposition 2. If $\theta \geq \hat{\theta}(0)$, the amount of seed money to guarantee that agents with signals larger than 0 choose Donate is sufficient. If $\theta<\hat{\theta}(0)$, we need enough seed money $\ell$ so that the shifted threshold $c-\ell$ is weakly smaller than the possible total amount (i.e., $\left.c-\ell \leq \int_{0}^{\infty} x(v) f(v ; \theta) d v\right)$.

Next, we consider the minimal seed money for success. The project is not a success without seed money if it is not efficient and $\theta<\hat{\theta}\left(k^{*}(0)\right)$. In this case, in order to achieve success, we have the following proposition.

Proposition 6. The minimal amount of seed money for success $\ell^{*}$ is such that if $\hat{\theta}(0) \leq \theta<\hat{\theta}\left(k^{*}(0)\right), \hat{\theta}\left(k^{*}\left(\ell^{*}\right)\right)=\theta$, and if $\theta<\hat{\theta}(0), \ell^{*}=c-$ $\int_{0}^{\infty} x(v) f(v ; \theta) d v$.

Note that from Remarks 3 and 6 , in the case of $\hat{\theta}(0) \leq \theta<\hat{\theta}\left(k^{*}(0)\right)$, $\hat{\theta}\left(k^{*}(\ell)\right)$ is continuously and strictly decreasing in $\ell \in[0, \bar{\ell}]$. Thus $\ell^{*}$ is 
uniquely determined. In this case, the minimal seed money for success is strictly smaller than that for efficiency, and this suits our intuition. In Example 2, in the case of $\theta=0$, both $\bar{\ell}=\ell^{*}=2.01058$. However, in the case of $\theta=2, \bar{\ell}=2.01058$ and $\ell^{*}=1.01058$.

To calculate the minimal seed money for efficiency and success, a charity fund-raiser needs to know the actual mean of the distribution $\theta$. Indeed, the collection of the actual signal distribution is quite difficult for a charity fund-raiser. It involves a considerable cost for preliminary surveys on the potential donors to approach the actual mean $\theta$, and it is very beneficial.

\section{Discussion}

In the previous section, we establish Proposition 4, which is quite compatible with the empirical result in List and Lucking-Reiley (2002). In this section, we discuss the still remaining inconsistency of our model with List and Lucking-Reiley (2002). In our model, the per-capita amount of donations strictly decreases along with the increase of seed money, whereas the percapita amount of donations strictly increases corresponding to the increase of seed money in List and Lucking-Reiley (2002). We discuss the source of this inconsistency and propose some ideas for its resolution.

In the previous section, Example 2 demonstrates that the per-capita amount of donation is strictly decreasing with the amount of seed money. The mechanism is quite simple. We assume that the amount of donation $x\left(v_{i}\right)$ of agent $i$ with signal $v_{i}$ is exogenously given and never changes to the amount of seed money. From Remark 6, if the amount of seed money increases, the threshold signal $k^{*}(\ell)$ decreases. Note that an agent who changes her action from Not Donate to Donate because of the increase in seed money has a smaller signal and a smaller donation than agents who continue to choose to Donate. This induces the per-capita amount of donation to decrease.

To remove this inconsistency in the extended version of our model, we need to increase $x\left(v_{i}\right)$ according to the increase of seed money. Two approaches are considerable for this purpose.

The first approach is to render $x\left(v_{i}\right)$ endogenously determined as an optimal value corresponding to the probability of success. This maintains the assumption that the effect of seed money is only the threshold shift effect. As the probability of success depends on the amount of donation obtained from other agents, this optimization problem is highly complicated. At present, we are unsure about whether this approach makes sense.

The second approach entails the introduction of the quality signal effect of seed money. The increase of $x\left(v_{i}\right)$ corresponding to the increase in seed money can be considered as the consequence of the quality signal effect. For example, consider the simple introduction of the quality signal effect 
as follows. Let $\theta$ be the mean of each agent's signal, similarly to Section 2. Let $\theta:[0, c) \rightarrow \mathbb{R}$ be a strictly increasing and continuous function such that $\theta(0)=\theta$. Assume that when seed money $\ell$ is granted, the mean of signal $\theta$ is shifted to $\theta(\ell)$. Moreover, assume that the standard deviation $\sigma$ remains the same. We interpret this shift of $\theta$ to $\theta(\ell)$ as indicating that an agent $i$ receiving a signal $v_{i}$ when no seed money is granted receives a signal $v_{i}(\ell)=v_{i}+(\theta(\ell)-\theta)$ when seed money $\ell$ is granted. It is obvious that in this model, the inconsistency regarding the per-capita amount of donation is resolved.

The second explanation also suggests the possibility of constructing a model in which the threshold shift effect and the quality signal effect are complementary. Which effect actually works remains an interesting question in both the theoretical and empirical work.

\section{Concluding Remarks}

In this paper, we construct a global coordination game model of charitable giving. We show that by merely considering the threshold effect of seed money, the proportion of agents who decide to donate and the total amount of donations strictly and continuously increase according to the increase in seed money.

We construct a simultaneous model for comparison with the empirical work in List and Lucking-Reiley (2002). However, the actual capital campaigns of charities usually allow agents to donate in a certain period, and possibly represented by dynamic games. Even in dynamic models, our view that charitable donation is indeed a coordination game plays an important role. ${ }^{19}$ We hope that this paper encourages further study of donation behavior and thereby assists future charity projects.

\section{Appendix}

In the Appendix, we provide the proofs of the Propositions and Remarks. We omit the proofs of Remarks 1 and 4 since they are straightforward.

Proof of Remark 2. The continuity and differentiability of $p(\cdot)$ are obvious. Note that for $v_{i}>0$,

$$
p^{\prime}\left(v_{i}\right)=\frac{-x\left(v_{i}\right)+v_{i} x^{\prime}\left(v_{i}\right)}{v_{i}^{2}}
$$

\footnotetext{
${ }^{19}$ Famous dynamic models of investments in projects include Admati and Perry (1991) and Marx and Matthews (2000). Dynamic models of investment in projects in the global game framework include, for example, Heidhues and Melissas (2006), Dasgupta (2007), and Barbieri and Mattozzi (2008).
} 
Since $x(\cdot)$ is weakly concave in $v_{i}>0,-x\left(v_{i}\right)+v_{i} x^{\prime}\left(v_{i}\right) \leq 0$. It implies that for $v_{i}>0, p^{\prime}\left(v_{i}\right) \leq 0$, and $p\left(v_{i}\right)$ is weakly decreasing in $v_{i} \geq 0$.

Proof of Remark 3. Remember that $\int_{\hat{\theta}\left(v_{i}\right)}^{\infty} f\left(v ; v_{i}\right) d v=p\left(v_{i}\right)$ for $v_{i}>0$. Since $p(\cdot)$ is weakly decreasing in $v_{i} \geq 0$ by Remark 2 , for $v_{i}^{\prime}>v_{i}>0$,

$$
\int_{\hat{\theta}\left(v_{i}\right)}^{\infty} f\left(v ; v_{i}\right) d v=p\left(v_{i}\right) \geq p\left(v_{i}^{\prime}\right)=\int_{\hat{\theta}\left(v_{i}^{\prime}\right)}^{\infty} f\left(v ; v_{i}^{\prime}\right) d v .
$$

Thus the continuity of $p(\cdot)$ implies (i) $\hat{\theta}\left(v_{i}\right)$ is strictly increasing and continuous in $v_{i} \geq 0$.

Note that if $p\left(v_{i}\right)=p\left(v_{i}^{\prime}\right), \hat{\theta}\left(v_{i}^{\prime}\right)-\hat{\theta}\left(v_{i}\right)=v_{i}^{\prime}-v_{i}$ and if $p\left(v_{i}\right)>p\left(v_{i}^{\prime}\right)$, $\hat{\theta}\left(v_{i}^{\prime}\right)-\hat{\theta}\left(v_{i}\right) \geq v_{i}^{\prime}-v_{i}$. (See Figure 2 for an illustration.) They imply (ii) for $v_{i}^{\prime}>v_{i} \geq 0, \hat{\theta}\left(v_{i}^{\prime}\right)-\hat{\theta}\left(v_{i}\right) \geq v_{i}^{\prime}-v_{i}$.

Since $\hat{\theta}(0) \in \mathbb{R}$, the construction of $\hat{\theta}\left(v_{i}\right)$ as $\int_{\hat{\theta}\left(v_{i}\right)}^{\infty} f\left(v ; v_{i}\right) d v=p\left(v_{i}\right)$ implies (iii) $\lim _{v \rightarrow+\infty} \hat{\theta}(v)=+\infty$.

[Figure 2 about here.]

Proof of Remark 5. Let $v^{\prime}>v>0$. Since $\hat{\theta}\left(v_{i}^{\prime}\right)-\hat{\theta}\left(v_{i}\right) \geq v_{i}^{\prime}-v_{i}$ by Remark $3, \int_{v_{i}}^{\infty} f\left(v ; \hat{\theta}\left(v_{i}\right)\right) d v \leq \int_{v_{i}^{\prime}}^{\infty} f\left(v ; \hat{\theta}\left(v_{i}^{\prime}\right)\right) d v$. Since $x(\cdot)$ is a strictly increasing function, it implies that

$$
\int_{v_{i}}^{\infty} x(v) f\left(v ; \hat{\theta}\left(v_{i}\right)\right) d v<\int_{v_{i}^{\prime}}^{\infty} x(v) f\left(v ; \hat{\theta}\left(v_{i}^{\prime}\right)\right) d v .
$$

We have the statement of the remark.

Proof of Proposition 1. Proposition 2.1 of Morris and Shin (2003) provides the equilibrium of a general global game where the (ex-post) payoff of an agent depends on her action, the proportion of other agents' strategies, and her own signal. Even though in our game $G$, the payoff of an agent depends on her action, the total amount of donation, and her own signal, our Proposition 1 is essentially the same as their Proposition 2.1. We owe Morris and Shin (2003) and originally Carlsson and van Damme (1993) their proof techniques.

Case 1: $\int_{0}^{\infty} x(v) f(v ; \hat{\theta}(0)) d v<c$.

(A) First, we construct a sequence of recursive values $\bar{v}^{0}, \bar{v}^{1}, \bar{v}^{2}, \cdots$ such that for agents with signals larger than each value, Not Donate is the interim strictly dominated action.

By Assumption 1, for any agent $i$ with $v_{i}>V$, Donate is the strictly dominant action, and Not Donate is strictly dominated. Let $\bar{v}^{0}=V$. 
Let $t \in \mathbb{N} \cup\{0\}$. Assume as an induction hypothesis that for any agent $i$ with $v_{i}>\bar{v}^{t}$, Not Donate is the interim strictly dominated action, and they choose to Donate. Let $\bar{v}^{t+1}$ be such that

$$
\int_{\bar{v}^{t}}^{\infty} x(v) f\left(v ; \hat{\theta}\left(\bar{v}^{t+1}\right)\right) d v=c .
$$

Suppose, at first, that all the other agents with signals smaller than $\bar{v}^{t}$ choose Not Donate. Then, (13) represents that if the mean signal is $\hat{\theta}\left(\bar{v}^{t+1}\right)$, the total amount of donation equals $c$. Thus if the mean signal is larger than $\hat{\theta}\left(\bar{v}^{t+1}\right)$, the project is a success.

Note that for an agent $i$ with $v_{i}>\bar{v}^{t+1}, \operatorname{Pr}\left(\theta \geq \hat{\theta}\left(\bar{v}^{t+1}\right)\right)>\operatorname{Pr}(\theta \geq$ $\left.\hat{\theta}\left(v_{i}\right)\right)=p\left(v_{i}\right)$ since $\hat{\theta}\left(v_{i}\right)>\hat{\theta}\left(\bar{v}^{t+1}\right)$. This means that from $i$ 's point of view, the probability of success is larger than $p\left(v_{i}\right)$, and Donate is more profitable than Not Donate.

Notice that if we drop the supposition that all other agents with signals smaller than $\bar{v}^{t}$ choose Not Donate, the probability of success for $i$ with $v_{i}>\bar{v}^{t+1}$ is higher than that with the supposition. Thus, for $i$ with $v_{i}>\underline{v}^{t+1}$, Not Donate is the interim strictly dominated action.

Next, we show that $\kappa \leq \bar{v}^{t+1} \leq \underline{v}^{t} \leq V$.

First, notice $\kappa \leq V=\bar{v}^{0}$ by Assumption 2 and Remark 5 .

Assume as an induction hypothesis that $\kappa \leq \bar{v}^{t}$. Then $\int_{\kappa}^{\infty} x(v) f(v ; \hat{\theta}(\kappa)) d v=$ $c$ by the definition of $\kappa$ and Remark 5 imply that

$$
\int_{\bar{v}^{t}}^{\infty} x(v) f\left(v ; \hat{\theta}\left(\bar{v}^{t}\right)\right) d v \geq c .
$$

(13), (14) and Remark 4 imply that $\bar{v}^{t} \geq \bar{v}^{t+1}$. Similarly, since $\kappa \leq \bar{v}^{t}$, $\int_{\kappa}^{\infty} x(v) f(v ; \hat{\theta}(\kappa)) d v=c,(13)$ and Remark 4 imply $\bar{v}^{t+1} \geq \kappa$. Hence $\kappa \leq$ $\bar{v}^{t+1} \leq \underline{v}^{t} \leq V$.

By recursive means, we have that the sequence $\bar{v}^{0}, \bar{v}^{1}, \bar{v}^{2}, \cdots$ is weakly decreasing and has an lower bound $\kappa$. Thus it has the limit value. Let $\bar{v}$ be the limit value. (i.e., $\lim _{t \rightarrow+\infty} \bar{v}^{t}=\bar{v}$.) Since (8) is continuous in both $k$ and $v_{i}$ by Remark 4, we have that $\int_{\bar{v}}^{\infty} x(v) f(v ; \hat{\theta}(\bar{v})) d v=c$. This implies $\bar{v}=\kappa$.

(B) Similarly, we can construct a sequence of recursive values $\underline{v}^{0}, \underline{v}^{1}, \underline{v}^{2}, \cdots$ such that for agents with signals smaller than each value, Donate is the interim strictly dominated action.

Let $\underline{v}^{0}=0$. Given $t \in \mathbb{N} \cup\{0\}$, let $\underline{v}^{t+1}$ be such that

$$
\int_{\underline{v}^{t}}^{\infty} x(v) f\left(v ; \hat{\theta}\left(\underline{v}^{t+1}\right)\right) d v=c .
$$

Then, Remark 1 and the symmetric reasoning of (A) guarantee that for agents with signals smaller than each value, Donate is the interim strictly dominated action. 
Similarly to (A), we have that $0 \leq \underline{v}^{t}<\underline{v}^{t+1}<\kappa$ for any $t \in \mathbb{N} \cup\{0\}$, and $\lim _{t \rightarrow+\infty} \underline{v}^{t}=\kappa$.

By discussions (A) and (B), we have the statement of Case 1.

Case $2: \int_{0}^{\infty} x(v) f(v ; \hat{\theta}(0)) d v \geq c$.

(A) We can use the same reasoning as (A) in Case 1 with only one modification. Instead of (13), let

$$
\bar{v}^{t+1}= \begin{cases}0 & \text { if } \int_{\bar{v}^{t}}^{\infty} x(v) f(v ; \hat{\theta}(0)) d v \geq c \\ w^{t+1} & \text { otherwise }\end{cases}
$$

where $w^{t+1} \in \mathbb{R}_{+}$is such that $\int_{\bar{v}^{t}}^{\infty} x(v) f\left(v ; \hat{\theta}\left(w^{t+1}\right)\right) d v=c$.

Then we have that $0 \leq \bar{v}^{t+1} \leq \underline{v}^{t} \leq V$ for any $t \in \mathbb{N} \cup\{0\}$, and $\lim _{t \rightarrow+\infty} \bar{v}^{t}=0$.

(B) Since Remark 1 says that for any agent $i$ with $v_{i}<0$, Not Donate is a strictly dominant action.

By discussions (A) and (B), we have the statement for Case 2.

Proof of Proposition 2. The if part is obvious. The only if part is explained in the paragraph after this proposition.

Proof of Proposition 3. if part : It is obvious that efficiency implies project success. The explanation for the case of $\theta \geq \hat{\theta}\left(k^{*}\right)$ is given in the paragraph after this proposition. only if part : We show the contraposition. Suppose $\theta<\hat{\theta}\left(k^{*}\right)$ and $\left(k^{*}>0\right.$ or $\left.\int_{0}^{\infty} x(v) f(v ; \theta) d v<c\right)$. If $\int_{0}^{\infty} x(v) f(v ; \theta) d v<c$, the project is never a success. If $\theta<\hat{\theta}\left(k^{*}\right)$ and $k^{*}>0$, then $\int_{k^{*}}^{\infty} x(v) f\left(v ; \hat{\theta}\left(k^{*}\right)\right) d v=$ $c>\int_{k^{*}}^{\infty} x(v) f(v ; \theta) d v$. This induces project failure.

Proof of Remark 6. In the case of $\ell \geq \underline{\ell}, k^{*}(\ell)=0$ is directly induced from Corollary 1. We consider the case of $\ell<\underline{\ell}$. Let a small $\epsilon>0$. Then, from Corollary 1, we have

$$
\int_{k^{*}(\ell)}^{\infty} x(v) f\left(v ; \hat{\theta}\left(k^{*}(\ell)\right)\right) d v=c-\ell
$$

and

$$
\int_{k^{*}(\ell+\epsilon)}^{\infty} x(v) f\left(v ; \hat{\theta}\left(k^{*}(\ell+\epsilon)\right)\right) d v=c-(\ell+\epsilon) .
$$

By subtracting both sides of (16) from those of (15), we have

$$
\int_{k^{*}(\ell)}^{\infty} x(v) f\left(v ; \hat{\theta}\left(k^{*}(\ell)\right)\right) d v-\int_{k^{*}(\ell+\epsilon)}^{\infty} x(v) f\left(v ; \hat{\theta}\left(k^{*}(\ell+\epsilon)\right)\right) d v=\epsilon>0 .
$$

Remark 5 and (17) imply $k^{*}(\ell)>k^{*}(\ell+\epsilon)$. Hence, $k^{*}(\ell)$ is strictly decreasing and continuous in $\ell<\underline{\ell}$. 
Proof of Proposition 4. Let $\ell<\underline{\ell}$. Note that, under this condition, the proportion of agents who decide to donate is represented by $\int_{k^{*}(\ell)}^{\infty} f(v ; \theta) d v$ and the total amount of donations is given by $\int_{k^{*}(\ell)}^{\infty} x(v) f(v ; \theta) d v$. Since both are strictly decreasing in $k^{*}(\ell)$, Remark 6 induces the statement.

Proof of Proposition 5. First consider the case of $\theta \geq \hat{\theta}(0)$. Let $\ell \geq \bar{\ell}$. Then $\int_{0}^{\infty} x(v) f(v ; \theta) d v \geq \int_{0}^{\infty} x(v) f(v ; \hat{\theta}(0)) d v \geq c-\ell$. This occasions project success and $k^{*}(\ell)=0$. Thus it is efficient. On the other hand, let $\ell^{\prime}<\bar{\ell}$. Then $\int_{0}^{\infty} x(v) f(v ; \hat{\theta}(0)) d v=c-\bar{\ell}<c-\ell^{\prime}$. Then $k^{*}\left(\ell^{\prime}\right)>0$ and this brings about inefficiency.

Next, we consider the other case, in which $\theta<\hat{\theta}(0)$. Let $\ell \geq \bar{\ell}$. Then $\int_{0}^{\infty} x(v) f(v ; \hat{\theta}(0)) d v \geq \int_{0}^{\infty} x(v) f(v ; \theta) d v \geq c-\ell$. This occasions project success and $k^{*}(\ell)=0$. Thus it is efficient. Let $\ell^{\prime}<\bar{\ell}$. Then $\int_{0}^{\infty} x(v) f(v ; \theta) d v=c-\bar{\ell}<c-\ell^{\prime}$. This occasions project failure and inefficiency.

Proof of Proposition 6. First consider the case of $\hat{\theta}(0) \leq \theta<\hat{\theta}\left(k^{*}\left(\ell^{*}\right)\right)$. Let $\ell \geq \ell^{*}$. Remark 6 implies that $k^{*}(\ell) \leq k^{*}\left(\ell^{*}\right)$. Thus we have $\hat{\theta}\left(k^{*}(\ell)\right) \leq$ $\hat{\theta}\left(k^{*}\left(\ell^{*}\right)\right)=\theta$. Since Corollary 1 states that

$$
\int_{k^{*}(\ell)}^{\infty} x(v) f\left(v ; \hat{\theta}\left(k^{*}(\ell)\right)\right) d v=c-\ell
$$

$\hat{\theta}\left(k^{*}(\ell)\right) \leq \theta$ implies that

$$
\int_{k^{*}(\ell)}^{\infty} x(v) f(v ; \theta) d v \geq c-\ell
$$

This occasions project success. Let $\ell^{\prime}<\ell^{*}$. Then the symmetric reasoning brings $\int_{k^{*}\left(\ell^{\prime}\right)}^{\infty} x(v) f(v ; \theta) d v<c-\ell^{\prime}$. This induces project failure.

For the case of $\theta<\hat{\theta}(0)$, the proof is exactly the same as for the case of $\theta<\hat{\theta}(0)$ in Proposition 5.

\section{References}

Admati, A. R., Perry, M., 1991. Joint Projects without Commitment. Review of Economic Studies 58 (2), 259-276.

Andreoni, J., 1988. Privately Provided Public Goods in a Large Economy: The Limits of Altruism. Journal of Public Economics 35 (1), 57-73.

Andreoni J., 1989. Giving with Impure Altruism: Applications to Charity and Ricardian Equivalence. Journal of Political Economy 97 (6), 14471458 . 
Andreoni, J., 1990. Impure Altruism and Donations to Public Goods: A Theory of Warm-Glow Giving. Economic Journal 100 (401), 464-477.

Andreoni, J., 1998. Toward a Theory of Charitable Fund-Raising. Journal of Political Economy 106 (6), 1186-1213.

Andreoni, J., 2006a. Leadership Giving in Charitable Fund-Raising. Journal of Public Economic Theory 8 (1), 1-22.

Andreoni, J., 2006b. Philanthropy. in Handbook of the Economics of Giving, Altruism and Reciprocity Volume 2: Applications, edited by Kolm, S. -C., Ythier, J. M. North Holland, Amsterdam.

Bac, M., Bag, P. K., 2003. Strategic Information Revelation in Fund-Raising. Journal of Public Economics 87 (3-4), 659-679.

Bag, P. K., Roy, S., 2008. Repeated Charitable Contributions under Incomplete Information. Economic Journal 118 (525), 60-91.

Barbieri, S., Mattozzi, A., 2009. Membership in Citizen Groups. Games and Economic Behavior 67 (1), 217-232.

Carlsson, H., van Damme, E., 1993. Global Games and Equilibrium Selection. Econometrica 61 (5), 989-1018.

Dasgupta, A., 2007. Coordination and Delay in Global Games. Journal of Economic Theory 134 (1), 195-225.

Giving USA Foundation, 2006. Giving USA 2006: the Annual Report on Philanthropy for the Year 2005. Giving USA Foundation.

Goldstein, I., Pauzner, A., 2005. Demand-Deposit Contracts and the Probability of Bank Runs. Journal of Finance 60 (3), 1293-1327.

Gradstein, M., 1994. Efficient Provision of a Discrete Public Good. International Economic Review 35 (4), 877-897.

Heidhues, P., Melissas, N., 2006. Equilibria in a Dynamic Global Game: the Role of Cohort Effects. Economic Theory 28 (3), 531-557.

List, J. A., Lucking-Reiley, D., 2002. The Effects of Seed Money and Refunds on Charitable Giving: Experimental Evidence from a University Capital Campaign. Journal of Political Economy 110 (1), 215-233.

List, J. A., Rondeau, D., 2003. The Impact of Challenge Gifts on Charitable Giving: an Experimental Investigation. Economics Letters 79 (2), 153159 .

Marx, L. M., Matthews, S., 2000. Dynamic Voluntary Contribution to a Public Project. Review of Economic Studies 67 (2), 327-358. 
Morris, S., Shin, H. S., 1998. Unique Equilibrium in a Model of Self-Fulfilling Attacks. American Economic Review 88 (3), 587-597.

Morris, S., Shin, H. S., 2003. Global Games: Theory and Applications. in Advances in Economics and Econometrics (Proceedings of the Eighth World Congress of the Econometric Society), edited by Dewatripont, M., Hansen, L., Turnovsky, S. Cambridge University Press, Cambridge.

Morris, S., Shin, H. S., 2004. Coordination Risk and the Price of Debt. European Economic Review 48 (1), 133-153.

Palfrey, T. R., Prisbrey, J. E., 1996. Altruism, Reputation and Noise in Linear Public Goods Experiments. Journal of Public Economics 61 (3), 409-427.

Palfrey, T. R., Prisbrey, J. E., 1997. Anomalous Behavior in Public Goods Experiments: How Much and Why? American Economic Review 87 (5), 829-846.

Palfrey, T. R., Rosenthal, H., 1984. Participation and the Provision of Discrete Public Goods: a Strategic Analysis. Journal of Public Economics 24 (2), 171-193.

Potters, J., Sefton, M., Vesterlund, L., 2005. After You - Endogenous Sequencing in Voluntary Contribution Games. Journal of Public Economics 89 (8), 1399-1419.

Ribar, D. C., Wilhelm, M. O., 2002. Altruistic and Joy-of-giving Motivations in Charitable Behavior. Journal of Political Economy 110 (2), 425-457.

Romano, R., Yildirim, H., 2001. Why Charities Announce Donations: a Positive Perspective. Journal of Public Economics 81 (3), 423-447.

Vesterlund, L., 2003. The Informational Value of Sequential Fundraising. Journal of Public Economics 87 (3-4), 627-657. 
Table1: Results of the Field Experiment by List and Lucking-Reiley (2002)

\begin{tabular}{lccc}
\hline A. Experimental Design & & & \\
\hline Number of solicitations mailed & 500 & 500 & 500 \\
Seed money (\%) & $10 \%$ & $33 \%$ & $67 \%$ \\
Seed money (\$) & $\$ 300$ & $\$ 1,000$ & $\$ 2,000$ \\
\hline B. Result & & & \\
\hline Number of contributions & 17 & 33 & 42 \\
Participation rate & $3.40 \%$ & $6.60 \%$ & $8.40 \%$ \\
Total contributions & $\$ 202$ & $\$ 805$ & $\$ 1,485$ \\
Mean amount given & $\$ 11.88$ & $\$ 24.39$ & $\$ 35.36$ \\
Standard error of mean amount & $\$ 2.27$ & $\$ 2.50$ & $\$ 2.26$ \\
\hline
\end{tabular}

Source: Table 1 of List and Lucking-Reiley (2002) 
Table 2: the Results of Example 1

\begin{tabular}{ccccc}
\hline$\theta$ & proportion of donors & total donation & $\begin{array}{c}\text { mean amount of } \\
\text { donation }\end{array}$ & success/failure \\
\hline 5 & 0.538988 & 6.66530 & 12.3663 & $\mathrm{~s}$ \\
0 & 0.343800 & 3.67958 & 10.7027 & $\mathrm{f}$ \\
-5 & 0.183498 & 1.73830 & 9.47316 & $\mathrm{f}$ \\
-10 & 0.080441 & 0.68844 & 8.55843 & $\mathrm{f}$ \\
\hline
\end{tabular}


Table 3: the Results of Example 2

\begin{tabular}{ccccccc}
\hline$\theta$ & $\ell$ & $k^{*}$ & $\begin{array}{c}\text { proportion of } \\
\text { donors }\end{array}$ & total donation & $\begin{array}{c}\text { mean amount } \\
\text { of donation }\end{array}$ & success /failure \\
\hline 2 & 0 & 4.02115 & 0.419913 & 4.74859 & 11.30851 & $\mathrm{f}$ \\
& 1 & 2.02115 & 0.499156 & 4.98773 & 9.99233 & $\mathrm{f}$ \\
& 2 & 0.02115 & 0.578432 & 5.06894 & 8.76324 & $\mathrm{~s}$ \\
& 3 & 0 & 0.57926 & 5.06895 & 8.75073 & $\mathrm{~s}$ \\
& 0 & 4.02115 & 0.343800 & 3.67958 & 10.7027 & $\mathrm{f}$ \\
& 1 & 2.02115 & 0.419913 & 3.90876 & 9.30850 & $\mathrm{f}$ \\
& 2 & 0.02115 & 0.499156 & 3.98941 & 7.99231 & $\mathrm{f}$ \\
& 3 & 0 & 0.500000 & 3.98942 & 7.97884 & $\mathrm{~s}$ \\
\hline
\end{tabular}




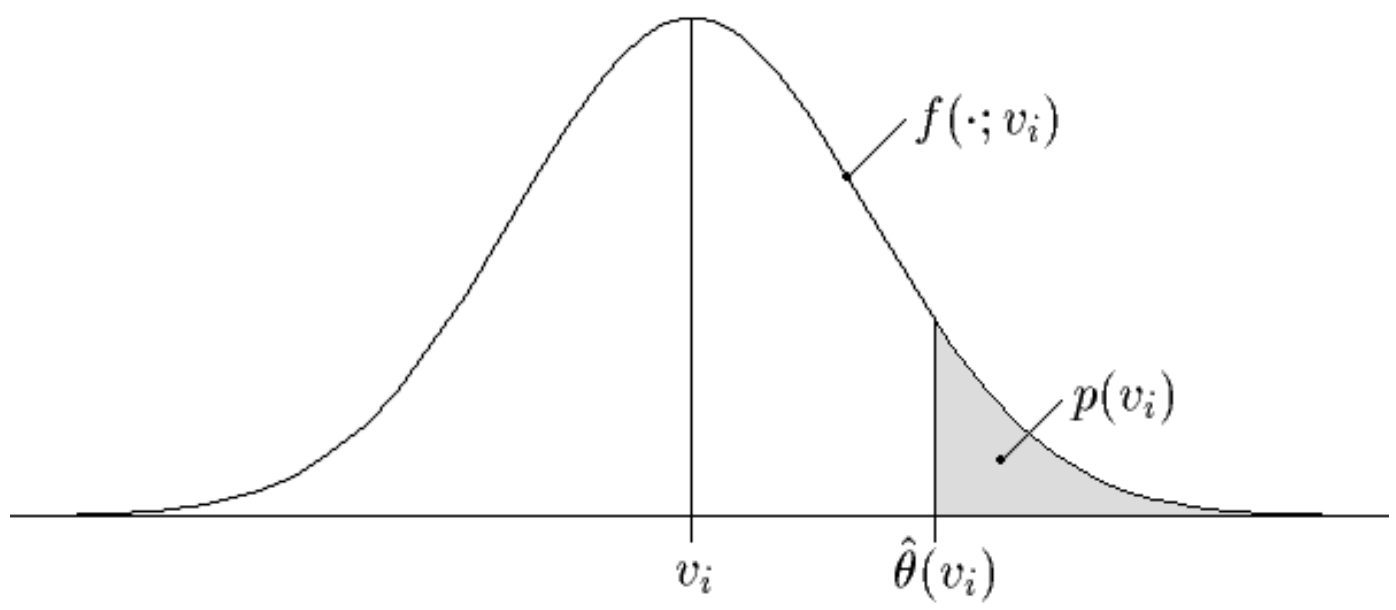

Figure 1: The relationship between $v_{i}, p\left(v_{i}\right)$, and $\hat{\theta}\left(v_{i}\right)$ 


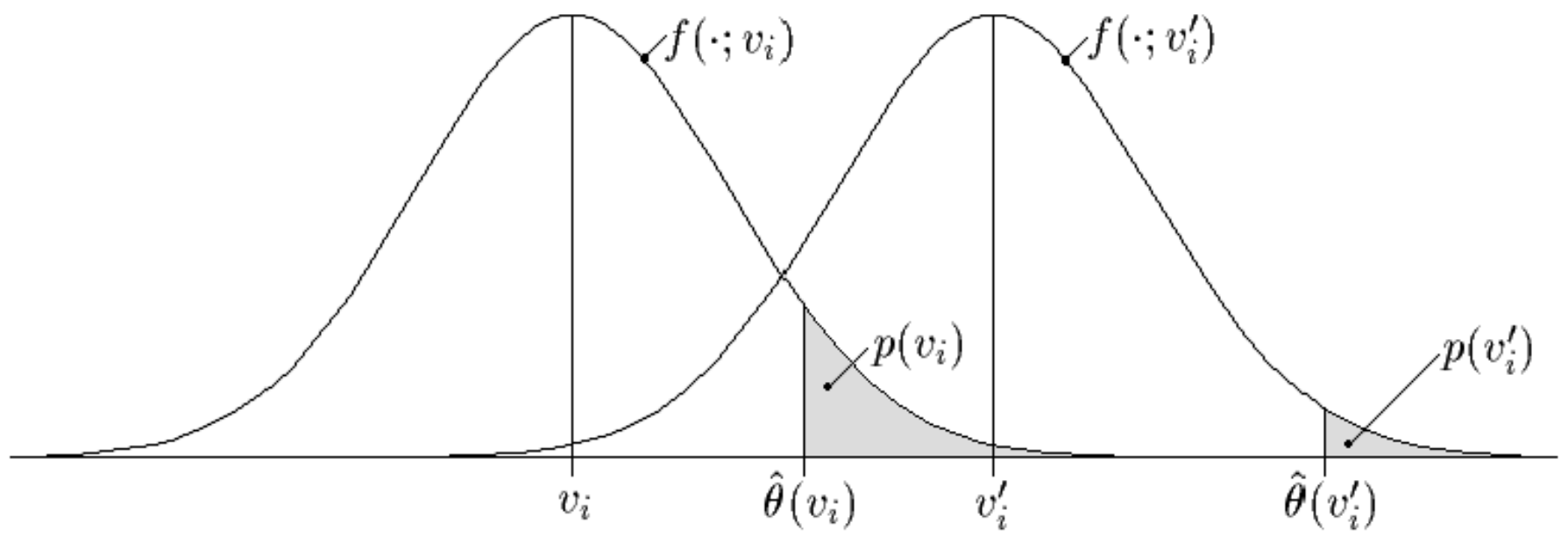

Figure 2: Illustration for the proof of Remark 3 


\title{
Supplementary Note for "A Coordination Game Model of Charitable Giving and Seed Money Effect"
}

\author{
Kentaro Hatsumi*
}

This Version: September 15, 2009

In this supplementary note, (i) we first show that some alternative payoff structures other than that in Payoff Tables (3) and (4) are available with the same consequences. (ii) Next, we show that without Assumptions 1 and 2, the equilibrium in Proposition 1 can be derived as the unique Bayesian Nash equilibrium under the symmetric switching strategy assumption.

(i) In the case of $v_{i}>0$, instead of Payoff Table (3), we assume that the payoff table is

\begin{tabular}{l|cc} 
& Success & Failure \\
\hline Donate & $v_{i}-x\left(v_{i}\right)$ & $-x\left(v_{i}\right)$ \\
Not Donate & $b\left(v_{i}\right)$ & 0
\end{tabular}

where $b: \mathbb{R}_{+} \rightarrow \mathbb{R}_{+}$is a differentiable function such that for all $v>0$, $0 \leq b(v) \leq v-x(v), b^{\prime}(v) \geq 0,\{b(v) / x(v)\}^{\prime} \leq 0$, and there exists $\beta \in(0,1)$ with $\lim _{v \rightarrow 0} b^{\prime}(v)=\beta$.

To derive the equivalent result to Proposition 1, it is sufficient to show the equivalent result to Remark 2 because this is the key to the uniqueness of the equilibrium.

Given Payoff Table (18), an agent $i$ with a positive signal $v_{i}>0$ chooses to donate if

$$
\begin{aligned}
& \operatorname{Pr}(\text { Success })\left(v_{i}-x\left(v_{i}\right)\right)+\left(1-\operatorname{Pr}(\text { Success })\left(-x\left(v_{i}\right)\right)>\operatorname{Pr}(\operatorname{Success}) b\left(v_{i}\right)\right. \\
\Longleftrightarrow & \operatorname{Pr}(\text { Success })>\frac{x\left(v_{i}\right)}{v_{i}-b\left(v_{i}\right)} .
\end{aligned}
$$

Since $\lim _{v_{i} \rightarrow 0} x\left(v_{i}\right)=0, \lim _{v_{i} \rightarrow 0} v_{i}-b\left(v_{i}\right)=0, \lim _{v_{i} \rightarrow 0} x^{\prime}\left(v_{i}\right)=\alpha$ and $\lim _{v \rightarrow 0} b^{\prime}(v)=\beta$, we have

$$
\lim _{v_{i} \rightarrow 0} \frac{x\left(v_{i}\right)}{v_{i}-b\left(v_{i}\right)}=\frac{\alpha}{1-\beta} \quad \text { (by l'Hospital's rule.) }
$$

*Corresponding to: Institute of Social and Economic Research, Osaka University. 6-1, Mihogaoka, Ibaraki, 567-0047, Japan. Email: hatsumi at iser.osaka-u.ac.jp 
Let a function $\bar{p}: \mathbb{R}_{+} \rightarrow \mathbb{R}_{++}$be such that

$$
\bar{p}\left(v_{i}\right)= \begin{cases}\frac{x\left(v_{i}\right)}{v_{i}-b\left(v_{i}\right)} & \text { if } v_{i}>0 \\ \frac{\alpha}{1-\beta} & \text { if } v_{i}=0 .\end{cases}
$$

Remark $2^{\prime} \cdot \bar{p}(\cdot)$ is weakly decreasing and continuous in $v_{i} \geq 0$.

Proof of Remark 2'. The continuity and differentiability of $p(\cdot)$ are obvious. Note that for $v_{i}>0$,

$$
\bar{p}^{\prime}\left(v_{i}\right)=\frac{-x\left(v_{i}\right)+v_{i} x^{\prime}\left(v_{i}\right)+x\left(v_{i}\right) b^{\prime}(v)-x^{\prime}\left(v_{i}\right) b(v)}{\left(v_{i}-b\left(v_{i}\right)\right)^{2}} .
$$

Since $x(\cdot)$ is weakly concave in $v_{i}>0,-x\left(v_{i}\right)+v_{i} x^{\prime}\left(v_{i}\right) \leq 0$. Furthermore, by $\{b(v) / x(v)\}^{\prime} \leq 0, x\left(v_{i}\right) b^{\prime}(v)-x^{\prime}\left(v_{i}\right) b(v) \leq 0$. These imply that for $v_{i}>0$, $p^{\prime}\left(v_{i}\right) \leq 0$, and $\bar{p}\left(v_{i}\right)$ is weakly decreasing in $v_{i} \geq 0$.

Because of Remark 2', for this alternative payoff setting, we can derive the equivalent results to Remarks 3 to 5 and Proposition 1 by using the same proof.

(ii) Next, we show that even without Assumptions 1 and 2, the strategy $s^{*}(\cdot)$ defined in Proposition 1 constitutes a Bayesian Nash equilibrium. To derive equilibrium, instead of Assumptions 1 and 2, we assume that agents take symmetric threshold strategies and these constitute common knowledge. We also show that $s^{*}(\cdot)$ is a unique Bayesian Nash equilibrium under these alternative assumptions.

Proposition 7. $s^{*}(\cdot)$ is a unique symmetric switching strategy constituting a Bayesian Nash equilibrium.

Proof of Proposition 7. First, we show that $s^{*}(\cdot)$ is a symmetric switching strategy constituting a Bayesian Nash equilibrium. Suppose that all agents except $i$ follow the strategy $s^{*}(\cdot)$. By the payoff matrix of this model, if $v_{i} \leq 0$, it is obvious that she has no incentive to donate. We focus on the case of $v_{i}>0$.

Case 1: $\int_{0}^{\infty} x(v) f(v ; \hat{\theta}(0)) d v \geq c$.

Note that for any $\theta \geq \hat{\theta}(0)$,

$$
\int_{0}^{\infty} x(v) f(v ; \theta) d v>\int_{0}^{\infty} x(v) f(v ; \hat{\theta}(0)) d v \geq c .
$$

This means that if the actual mean $\theta$ is larger than $\hat{\theta}(0)$ and all agents except $i$ follow the strategy $s^{*}(\cdot)$, the project is always a success. Also note that for any $v_{i}>0$,

$$
\int_{\hat{\theta}(0)}^{\infty} f\left(v ; v_{i}\right) d v>\int_{\hat{\theta}(0)}^{\infty} f(v ; 0) d v=p(0)
$$


This means that $i$ with a signal $v_{i}>0$ forecasts $\operatorname{Pr}(\theta>\hat{\theta}(0))>p(0)$.

Thus, (19) and (20) together imply that $i$ with a signal $v_{i}>0$ considers that the probability of project success is larger than $p(0)$, and she has an incentive to donate.

Case 2: $\int_{0}^{\infty} x(v) f(v ; \hat{\theta}(0)) d v<c$.

Note that for any $\theta>\hat{\theta}\left(k^{*}\right)$,

$$
\int_{k^{*}}^{\infty} x(v) f(v ; \theta) d v>\int_{k^{*}}^{\infty} x(v) f\left(v ; \hat{\theta}\left(k^{*}\right)\right) d v=c,
$$

and for any $\theta^{\prime} \leq \hat{\theta}\left(k^{*}\right)$,

$$
\int_{k^{*}}^{\infty} x(v) f\left(v ; \theta^{\prime}\right) d v \leq \int_{k^{*}}^{\infty} x(v) f\left(v ; \hat{\theta}\left(k^{*}\right)\right) d v=c .
$$

This means that under the assumption of that all agents except $i$ follow the strategy $s^{*}(\cdot)$, if the actual mean of the signals is larger than $\hat{\theta}\left(k^{*}\right)$, the project is a success, and if it is smaller than $\hat{\theta}\left(k^{*}\right)$, the project is a failure. Also note that for any $v_{i}>k^{*}$,

$$
\int_{\hat{\theta}\left(k^{*}\right)}^{\infty} f\left(v ; v_{i}\right) d v>\int_{\hat{\theta}\left(k^{*}\right)}^{\infty} f\left(v ; k^{*}\right) d v=p\left(k^{*}\right),
$$

and for any $v_{i}^{\prime} \leq k^{*}$,

$$
\int_{\hat{\theta}\left(k^{*}\right)}^{\infty} f\left(v ; v_{i}^{\prime}\right) d x \leq \int_{\hat{\theta}\left(k^{*}\right)}^{\infty} f\left(v ; k^{*}\right) d v=p\left(k^{*}\right) .
$$

This means that $i$ with a signal $v_{i}>k^{*}$ forecasts $\operatorname{Pr}\left(\theta>\hat{\theta}\left(k^{*}\right)\right)>p\left(k^{*}\right)$, and that with a signal $v_{i}^{\prime} \leq k^{*}$ forecasts $\operatorname{Pr}\left(\theta \leq \hat{\theta}\left(k^{*}\right)\right) \leq p\left(k^{*}\right)$.

Thus, (21) and (23) together imply that an agent $i$ with signal $v_{i}>k^{*}$ has an incentive to donate, and (22) and (24) together imply that an agent $i$ with signal $v_{i}^{\prime} \leq k^{*}$ has no incentive to donate.

In both Cases 1 and $2, s^{*}(\cdot)$ surely constitutes an Bayesian Nash equilibrium.

Next, we show the uniqueness of $s^{*}(\cdot)$. Case 1: $\int_{0}^{\infty} x(v) f(v ; \hat{\theta}(0)) d v \geq c$.

First, suppose, on the contrary, that a symmetric switching strategy $s(\cdot)$ with cutoff point $k>0$ constitutes a Bayesian Nash equilibrium. In this case, by Remark $5, \int_{k}^{\infty} x(v) f(v ; \hat{\theta}(k)) d v>c .{ }^{19}$ Thus, by that $\hat{\theta}(\cdot)$ is continuous and Remark 4, for a small $\epsilon>0$,

$$
\int_{k}^{\infty} x(v) f(v ; \hat{\theta}(k-\epsilon)) d v>c .
$$

\footnotetext{
${ }^{19}$ Note that to derive Remarks 1 to 5 , we do not need Assumptions 1 and 2.
} 
This means that if the realized mean of signals $\theta \geq \hat{\theta}(k-\epsilon)$ and any agent with a signal larger than $k$ donates, the project is a success. Note that

$$
\begin{array}{rlrl}
\int_{\hat{\theta}(k-\epsilon)}^{\infty} f\left(v ; k-\frac{\epsilon}{2}\right) d v & >\int_{\hat{\theta}(k-\epsilon)}^{\infty} f(v ; k-\epsilon) d v \\
& =p(k-\epsilon) & & (\text { by the definition of } \hat{\theta}(\cdot)) \\
& \geq p\left(k-\frac{\epsilon}{2}\right) . & & (\text { by Remark 2) }
\end{array}
$$

This means that an agent with signal $k-\frac{\epsilon}{2}$ predicts $\operatorname{Pr}(\theta \geq k-\epsilon)>p\left(k-\frac{\epsilon}{2}\right)$. Therefore, (25) and (26) together imply that an agent with signal $v_{i}=k-\frac{\epsilon}{2}$ has an incentive to donate. This is a contradiction.

Second, suppose, on the contrary, that a symmetric switching strategy $s(\cdot)$ with cutoff point $k<0$ constitutes a Bayesian Nash equilibrium. Immediately, an agent $i$ with a signal $k+\epsilon<0$ has an incentive not to donate. This is a contradiction.

Case 2: $\int_{0}^{\infty} x(v) f(v ; \hat{\theta}(0)) d v<c$.

First, suppose, on the contrary, that a symmetric switching strategy $s(\cdot)$ with a cutoff point $k>k^{*}$ constitutes a Bayesian Nash equilibrium. In this case, by $\int_{k^{*}}^{\infty} x(v) f\left(v ; \hat{\theta}\left(k^{*}\right)\right) d v=c$ and Remark $5, \int_{k}^{\infty} x(v) f(v ; \hat{\theta}(k)) d v>c$. We derive a contradiction similarly to Case 1 .

Second, suppose, on the contrary, that a symmetric switching strategy $s(\cdot)$ with a cutoff point $k<k^{*}$ constitutes a Bayesian Nash equilibrium.

In this case, by Remark $5, \int_{k}^{\infty} x(v) f(v ; \hat{\theta}(k)) d v<c$. Thus, by Remark 4 , for a small $\epsilon>0$,

$$
\int_{k}^{\infty} x(v) f(v ; \hat{\theta}(k+\epsilon)) d v<c
$$

This means that if the realized mean of the signals $\theta \leq \hat{\theta}(k+\epsilon)$ and any agent with a signal smaller than $k$ does not donate, the project is a failure. Note that

$$
\begin{array}{rlrl}
\int_{\hat{\theta}(k+\epsilon)}^{\infty} f\left(v ; k+\frac{\epsilon}{2}\right) d v & <\int_{\hat{\theta}(k+\epsilon)}^{\infty} f(v ; k+\epsilon) d v \\
& =p(k+\epsilon) & & (\text { by the definition of } \hat{\theta}(\cdot)) \\
& \leq p\left(k+\frac{\epsilon}{2}\right) . & & (\text { by Remark 2) }
\end{array}
$$

This means that an agent with signal $k+\frac{\epsilon}{2}$ predicts $\operatorname{Pr}(\theta \geq k+\epsilon)<p\left(k+\frac{\epsilon}{2}\right)$. Therefore, (27) and (28) together imply that an agent with signal $k+\frac{\epsilon}{2}$ has no incentive to donate. This is a contradiction.

By the above two cases, we have that $s^{*}(\cdot)$ with cutoff point $k^{*}$ is a unique switching strategy for a Bayesian Nash equilibrium. 